\title{
Nicht-relevante Metabolite in natürlichem Mineralwasser (2010/53)
}

Der Arbeitskreis Lebensmittelchemischer Sachverständiger der Länder und des Bundesamtes für Verbraucherschutz und Lebensmittelsicherheit (ALS) hat auf seiner 94. Sitzung am 08. und 09. Oktober 2009 in Dresden beschlossen, folgende Stellungnahme zu veröffentlichen:

Da die Problematik der nicht relevanten Metabolite in der Allgemeinen Verwaltungsvorschrift über die Anerkennung und Nutzungsgenehmigung von natürlichem Mineralwasser (AVV MTV) bisher nicht geregelt ist, sie sich aber in der Praxis stellt, vertritt der Arbeitskreis dazu folgende Auffassung: Sogenannte nicht-relevante Metabolite, die toxikologisch nicht relevant oder noch nicht bewertet sind, werden als anthropogene Verunreinigungen angesehen. Bei ihrem Vorhandensein ist die ursprüngliche Reinheit eines Mineralwassers nicht mehr gegeben. Die Bewertungsrichtlinie des UBA für Trinkwasser ist daher für natürliches Mineralwasser nicht anwendbar. Bezüglich der Beurteilung eines Nachweises von Spuren von Metaboliten wird auf den Orientierungswert für Pflanzenschutzmittelwirkstoffe in der Allgemeinen Verwaltungsvorschrift über die Anerkennung und Nutzungsgenehmigung von natürlichem Mineralwasser in Höhe von $0,05 \mu \mathrm{g} / \mathrm{l}$ verwiesen, der entsprechend zu berücksichtigen ist. 